\title{
Microwave ablation of hepatocellular carcinoma metastatic to peritoneum: diagnostic value of hydrodissection
}

\author{
Katayoun Samadi ${ }^{A, B, C, D}$, Ronald S. Arellano ${ }^{A, B, D, E, F}$ \\ Interventional Radiology Division, Radiology Department, Massachusetts General Hospital, United States
}

\section{Introduction}

Metastatic hepatocellular carcinoma (HCC) to the peritoneum is rare [1]. While microwave ablation (MWA) has an established role for the treatment HCC, its role for extrahepatic disease is less defined [2,3]. For tumours located on the surface of the liver this case demonstrates a successful use of MWA to treat an unusual manifestation of metastatic HCC. Hydrodissection is typically used to protect adjacent organs from thermal injury during ablation [4].

\section{Case description}

A 79-year-old man with a history of hepatitis B infection underwent laparoscopic left hepatectomy for a biopsy-proven 6.5-cm HCC. Four years later, surveillance magnetic resonance imaging (MRI) revealed a $1.2-\mathrm{cm}$

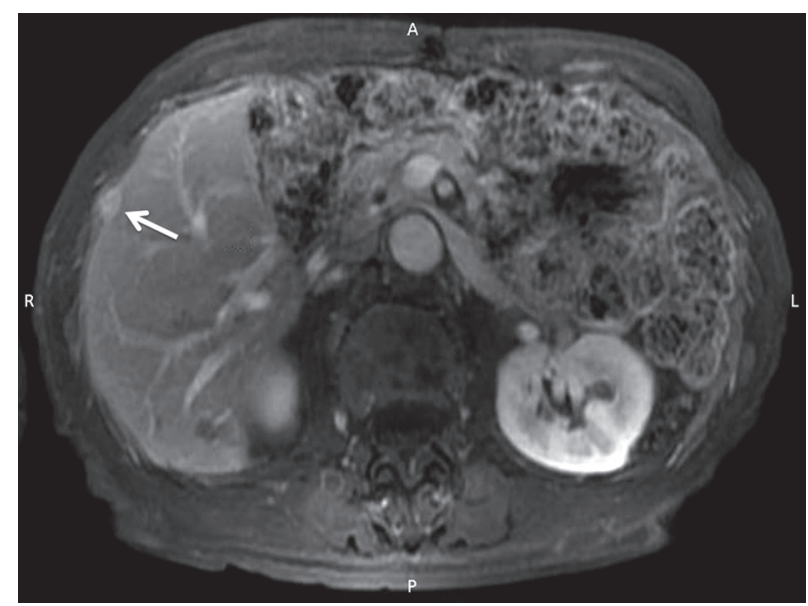

Figure 1. Gadolinium-enhanced MRI demonstrating an enhanced nodule on the periphery of the right hepatic lobe (white arrow) enhancing nodule; it was unclear from imaging whether the nodule was a surface implant or in the periphery of segment V (Figure 1). The patient was initially referred for surgical resection, but both the surgeon and patient were hesitant to embark on repeat operation. Therefore, the patient was referred to Interventional Radiology for percutaneous locoregional therapy. Ablative options of radiofrequency ablation, MWA, cryoablation, and irreversible electroporation were all considered. Ultimately, computed tomography (CT)-guided MWA was chosen based on the anticipated short ablation time.

Hydrodissection was performed in order to displace the liver edge from the peritoneal surface, in order to minimise thermal injury to the peritoneum. Using (CT) guidance, a 19-gauge Chiba needle was advanced into the peritoneal space, and $360 \mathrm{ml}$ of diluted contrast material

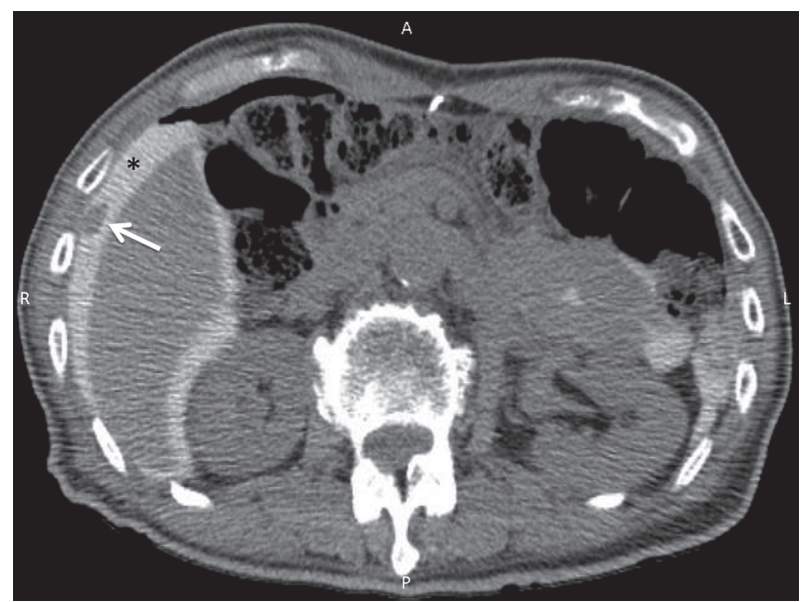

Figure 2. After hydrodissection, the nodule was identified as a peritoneal implant (white arrow). Black asterisk indicates hydrodissection fluid

Correspondence address:

Dr. Ronald S. Arellano, Interventional Radiology Division, Radiology Department, Massachusetts General Hospital, United States, e-mail: rarellano@mgh.harvard.edu Authors' contribution:

A Study design · B Data collection · C Statistical analysis · D Data interpretation · E Manuscript preparation · F Literature search · G Funds collection 


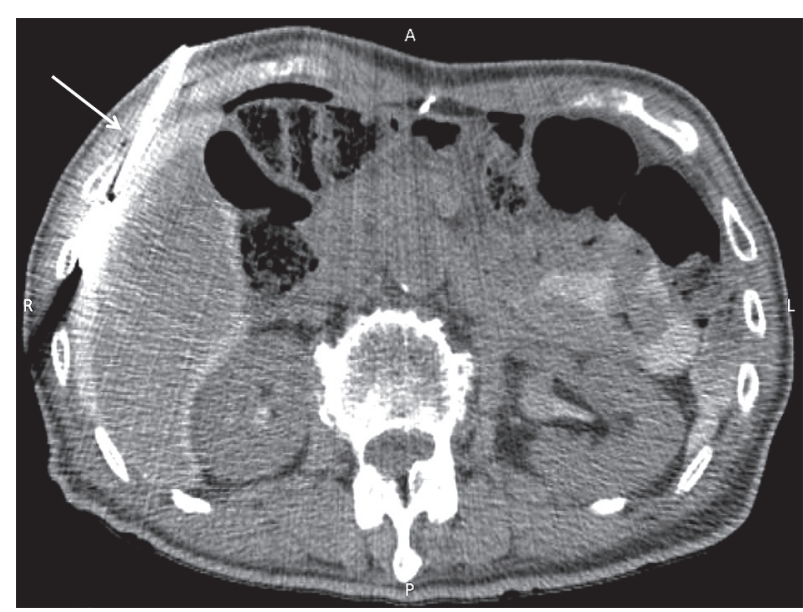

Figure 3. Axial CT image demonstrating microwave antenna (white arrow) in the peritoneal nodule. Microwave ablation was performed utilising $40 \mathrm{~W} \times 3$ minutes

(20 cc Ultravist diluted in 1000 cc of $0.9 \%$ NS) was injected. This revealed that the nodule was a peritoneal deposit (Figure 2). Subsequently, CT-guided MWA of the peritoneal nodule was performed using 40 watts for three minutes (Figure 3). An immediate post-ablation CT demonstrated a decrease in size of the lesion without immediate complication. A gadolinium-enhanced MRI obtained one month later showed no evidence of residual disease or peritoneal fluid accumulation (Figure 4).

\section{Conclusions}

In this case, hydrodissection served not only to displace the liver away from the peritoneum but also functioned as a diagnostic tool to clearly define the nodule location

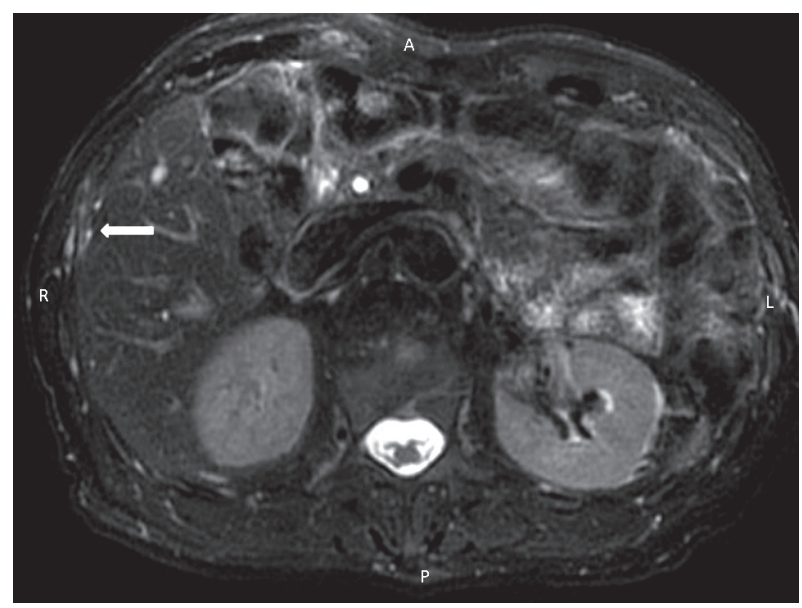

Figure 4. Gadolinium-enhanced T1-fat saturated MRI of the liver obtained one-month post MWA of peritoneal nodule. White arrow shows ablated area with no evidence of residual disease

as a peritoneal implant. As described in this paper, MWA could be a safe and technically effective method in the management of peritoneal seeding; however, additional studies are necessary to explore the potential value of MWA for extra-hepatic disease for HCC.

\section{Disclosure}

The Institutional Review Board approved the preparation of this paper.

\section{Conflict of interest}

The authors report no conflict of interest.

\section{References}

1. Terada T, Maruo H. Unusual extrahepatic metastatic sites from hepatocellular carcinoma. Int J Clin Exp Pathol 2013; 6: 816-820.

2. Shao H, Arellano RS. Percutaneous microwave ablation of hepatocellular carcinoma metastatic to a mesocolic lymph node. J Vasc Interv Radiol 2017; 28: 1281-1283.
3. Asvadi NH, Anvari A, Uppot RN, et al. CT-guided percutaneous microwave ablation of tumors in the hepatic dome: assessment of efficacy and safety. J Vasc Interv Radiol 2016; 27: 496-502.

4. Butros SR, DelCarmen MG, Uppot RN, Arellano RS. Image-guided thermal percutaneous thermal ablation of metastatic pelvic tumor from gynecologic malignancies. Obstet Gynecol 2014; 123: 500-505. 